\title{
ON THE RESPONSE OF VIBRATION ANALYSIS OF BEAM SUBJECTED TO MOVING FORCE
}

\section{AND MOVING MASS}

Usman, Mustapha Adewale ${ }^{1}$; Hammed, Fatai Akangbe ${ }^{2}$; Daniel, Deborah O. ${ }^{3}$; Okusaga, Sherrifat Taiwo $^{4}$; \& Badejo Oduyomi Michael

${ }^{2,4}$ Department of Mathematical Sciences, Olabisi Onabanjo University, Ago Iwoye, Nigeria.

Department of Computer Science, Southwestern University, Ogun State.

Department of Mathematical Sciences, Ondo State University of Science \& Technology, Okitipupa.

Email: usman.mustapha@oouagoiwoye.edu.ng

\section{ABSTRACT}

In this paper, vibration of beam subjected to moving force and moving mass is considered. Finite differential equation of order four. It was found that the response amplitude increases as the mass of the load increases for the case of moving mass while the response amplitude for the case of moving mass is not affected by increase in mass of the load. Also analysis shows that the response amplitude for the case of moving force is greater than that of moving mass.

KEYWORDS: Beam, Moving Mass, Moving Force, Finite Difference, Load, Mass, Concentrated \& Vibration

Accepted Date: 2 June, 2020

\section{INTRODUCTION}

Vibration is a mechanical phenomenon whereby oscillations (periodic or random) occur about an equilibrium point It is the motion of a particle or a body of system of connected bodies displaced from a place of equilibrium (Abu, 2003; Akinpelu, 2012; Awodola, 2005; Chen \& Jien, 2001). Beam is a structural element that is capable of withstanding load primarily by resisting bending. Beams are commonly found in many types of structures. Beams are characterized by their profile (shape of their cross section), their strength and material. Beams are typically made of steel, reinforced concrete or wood. They are also described by how they are supported, examples are simply supported beam, cantilever beam, overhanging beam and so on (Usman \& Muibi, 2019). Beams deflect when loaded and this must be limited to avoid damage and distress. For instance, large deflection in a steel beam supporting a partition could cause unacceptable cracking in the plaster (Gbadeyan \& Idowu, 2002; Gbadeyan \& Usman, 2003; Idowu et al., 2004; Ismail, 2011).

The purpose of vibrational analysis is to know the structural behavior under the influence of vart

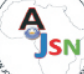

P-ISSN 2536-6904, E-ISSN 2705-2761 loads and to get the necessary information such as deformation, moments and dynamic forces (Kozien, 2013; Naguleswan, 2006).

A Simply-supported beam has a hinged connection at one end and roller connection at the other end. Vibration analysis of a Simply-supportedbeam is essential as it helps us to examine a number of real life systems. For example, in the automotive industry, the leaf spring suspension system study can be supposed to a Simply-supported beam analysis (Usman \& Adeboye, 2019). The numerical theory on Simply-supportedbeam has many applications for the calculations related to lon span railway and highway bridges. The moving load may be considered as either a moving force (without be conter or a moving mass (having (without ine conta effect) or a moving mass (having inertia effect). The vibration analysis of beam structure with moving load is a fundamenta problem in structural dynamics. The moving load varies in position as well as time. The problem of vibration of beam subjected to a moving load has been studied by many researchers (Usman, 2003).

\section{FORMULATION OF THE PROBLEM}

Consider a Simply-supported beam of finite length 
$\mathrm{L}$ subjected to a moving load, the differential equation for the deflection of the Simply-supported beam subjected to a moving load when the beam is of constant flexural rigidity EI is given (Gbadeyan \& Usman, 2006).

$E I W^{(i v)}(x, t)+\rho A W_{t t}(\mathrm{x}, \mathrm{t})=\mathrm{F}(\mathrm{x}, \mathrm{t})$

$E$ is the modulus of elasticity

$I$ is the constant moment of inertial of the beam's

$A$ cross section about the axis.

$W(x, t)$ is the deflection of the beam

$\rho$ is the density of the mass

$t$ is the mass

$x$ is the spatial coordinate and

$P(x, t)$ is the displaced force (i.e. the resultant concentrated force caused by moving mass). The displaced force per unit length $P(x, t)$ is defined as

$P(x, t)=F \delta(x-v t)$

Where $\mathrm{X}$ is the spatial function and $\mathrm{T}$ is the time function. To find the solution, finite Fourier sine transform method is adapted given by:

$f_{n}(t)=\int_{0}^{L} F(x, t) \sin \frac{n \pi}{L} x d x$

With the inverse

$\bar{F}(x, t)=\frac{2}{L} \sum_{n=1}^{\infty} f_{n}(t) \sin \frac{n \pi}{L} x$

We will now consider the solution for moving force and moving mass

If the inertial effect of mass is neglected, then the reacting force $M$ is given by Newton's second law reacting forch
which is

$F=-M g$ $\delta(\mathrm{x}-\mathrm{vt})=\left\{\begin{array}{ll}0 & \text { for } \mathrm{x}=0 \\ \infty & \text { for } \mathrm{x} \neq 0\end{array}\right\}$

With the property that

Substituting equation (2) into equation (1) we

$E I W^{(i v)}(x, t)+\rho A W_{t t}(x, t)=F \delta(x-V t)$

subjected to the following boundary conditions.

$w(0, t)=w(L, t)=0$

$W_{x x}(0, t)=W_{x x}(L, t)=0$

Finally, the initial conditions are:

$w(\bar{x}, 0)=w_{0}(x)=0$

$W_{t}(x, 0)=W_{0}(x)=0$

\section{Method of Solution}

We proceed to solve the above initial-boundary

value problem described by
Where $\delta(x-v t)$ is the dirac-delta function defined

$\int_{a}^{b} \delta(x-v t) f(x)=\left\{\begin{array}{cl}f(v, t) & a<v t<b \\ 0 & a<b<v t \\ 0 & v t<a<b\end{array}\right.$ have

For $0<v t<L, 0<x<L, F$

is the force due to gravity and $\mathrm{V}$ is the velocity of the load. The above governing equation is

$W(x, t)=X(x) T(t)$

$W(x, t)=\sum_{n=1}^{\infty} W_{n}(x, t)-\sum_{n=1}^{\infty} T_{n}(t) \sin \frac{n \pi}{L} x$

Find the function $f_{n}(t)$, where

$F(x, t)=M g \delta(x-v t)$

So we have

$f_{n}(t)=-2 g \frac{M}{L} \int_{0}^{L}\left[\delta(x-v t) \sin \frac{n \pi}{L} x\right] d x$

Applying the property of Dirac-delta function given in (3), we have

$f_{n}(t)=-2 g \frac{M}{L} \sin \frac{n \pi v}{L} t$

Substituting (15) into (11) after carrying out integration by using integration by part

$F(x, t)=-2 g \frac{M}{L} \sum_{n=1}^{\infty} \sin \frac{n \pi v}{L} t \sin \frac{n \pi}{L} x$

A $\mathrm{JSN}_{\mathrm{S}}$

P-ISSN 2536-6904, E-ISSN 2705-2761
Substituting (17) into (1) above, we have

$E I\left(\frac{n \pi}{L}\right)^{4} \sum_{n=1}^{\infty} T_{n}(t) \sin \frac{n \pi}{L} x+\rho A \sum_{n=1}^{\infty} T_{n}^{\prime \prime}(t) \sin \frac{n \pi}{L} x=-=-2 g \frac{M}{L} \sum_{n=1}^{\infty} \sin \frac{n \pi v}{L} t \sin \frac{n \pi}{L} x$

Divide through by

$\rho A \sum_{n=1}^{\infty} \sin \frac{n \pi}{L}$

We have

$T_{n}^{\prime \prime}(t)+\omega_{n}^{2} T_{n}(k)=k \sin \bar{\omega}_{n}(t)$

Where

$\omega_{n}^{2}=\frac{K I}{\rho A}\left(\frac{n \pi}{L}\right)^{4}, \bar{\omega}_{n}(t)=\frac{n \pi v}{L} \quad k=-\frac{2 M g}{\rho A L}$

Solving the homogeneous part, we have the solution of the form

$T_{A}(t)=G_{n} \sin \omega_{n}(t)+H_{n} \cos \omega_{n}(t)$

Using the method of undetermined coefficient, let

$T_{p}(t)=A_{n} \sin \bar{\omega}_{n}(t)+B_{n} \cos \bar{\omega}_{n}(t)$

Differentiating (21) and inserting back into (20), we have

$-\bar{\omega}_{n}(t) A_{n}-\bar{\omega}_{n}^{2}(t) B_{n} \cos \bar{\omega}_{n}(t)+\bar{\omega}_{n}^{2}(t) A_{n} \sin \bar{\omega}_{n}(t)+\bar{\omega}_{n}^{2} B_{n} \cos \bar{\omega}_{n}(t)$

$\sin \bar{\omega}_{n}(t): \quad-\bar{\omega}_{n} A_{n}+\bar{\omega}_{n}^{2} A_{n}=k$

$\cos \bar{\omega}_{n}(t): \quad-\bar{\omega}_{n} B_{n}+\bar{\omega}_{n}^{2} B_{n}=0$

Solve equations (23) and (24) simultaneously when $B_{n}=0$, from (23), we have that $A_{n}=\frac{k}{\left(\omega_{n}^{2}-\bar{\omega}_{n}^{2}\right)}$

Hence

$T_{p}(t)=\frac{k}{\left(\omega_{n}^{2}-\bar{\omega}_{n}^{2}\right)} \sin \bar{\omega}_{n}(t)$

So, from (21) and (26), we have

$T_{n}(t)=G_{n} \sin \omega_{n}(t)+H_{n} \cos \omega_{n}(t)+\frac{k}{\left(\omega_{n}^{2}-\bar{\omega}_{n}^{2}\right)} \sin \bar{\omega}_{n}(t)$

Substituting into the initial conditions as given in (8) and (9), so we have

$\sum_{n=1}^{\infty} T_{n}(0) \sin \frac{n \pi}{L} x=0$

and

$\sum_{n=1}^{\infty} T_{n}^{\prime}(0) \sin \frac{n \pi}{L} x^{\prime}=0$ 
$T_{n}(t)=G_{n} \sin \omega_{n}(t)+H_{n} \cos \omega_{n}(t)+\frac{k}{\left(\omega_{n}^{2}-\bar{\omega}_{n}^{2}\right)} \sin \bar{\omega}_{n}(t)$

$T_{n}^{\prime}(t)=G_{n} \cos \omega_{n}(t)-H_{n} \sin \omega_{n}(t)+\frac{k}{\left(\omega_{n}^{2}-\bar{\omega}_{n}^{2}\right)} \bar{\omega}_{n} \cos \bar{\omega}_{n}(t)$

Substituting the expression for $T_{n}(t)$ and $T_{n}{ }_{n}(t)$ in the initial conditions, we have $H_{n}=90$

$G_{n}=\frac{-k}{\bar{\omega}_{n}\left(\omega_{n}^{2}-\bar{\omega}_{n}^{2}\right)} \bar{\omega}_{n}$

So (27) becomes $T_{n}(t)=\frac{k}{\left(\omega_{n}^{2}-\bar{\omega}_{n}^{2}\right)}\left[\sin \omega_{n}(t)-R_{n} \sin \bar{\omega}_{n}(t)\right]$

where $R_{n}=\frac{\bar{\omega}_{n}}{\omega_{n}}$

Substituting (34) into (13), we have

$W(x, t)=\sum_{n=1}^{\infty} \frac{k}{\left(\omega_{n}^{2}-\bar{\omega}_{n}^{2}\right)}\left[\sin \omega_{n}(t)-R_{n} \sin \bar{\omega}_{n}(t)\right]$

Equation (35) is the deflection of beam subjected to moving force

\section{Moving Mass Problem}

Consider the inertial effect of moving mass, the reaction force $F$ displaced by mass $M$ is given by Newton's second law which is

$F=-M g-M \frac{\left.d^{2} W(x, t)\right|_{v t}}{d t^{2}}$

Here, $\frac{\left.d^{2} W(x, t)\right|_{v t}}{d t^{2}}$ is the transverse displacement of the load and $g$ is the acceleration due to gravity.

Substituting (36) into (2), we have

$P(x, t)=M\left(-g-\frac{\left.d^{2} W(x, t)\right|_{v t}}{d t^{2}}\right) \delta(x-v t)$

Substituting (37) into (1), the equation becomes

$E I W^{(i v)}(\lambda)(t)+\rho A W_{t t}(\lambda)=M\left(-g-\frac{\left.d^{2} W(x, t)\right|_{v t}}{d t^{2}}\right) \delta(x-v t)$

Where $W_{t t}(x, t)=W_{x t}(x, t)+2 V W_{x x}(x, t)+V^{2} W_{x t}(x, t)$

Substituting (39) into (38), we have

$E I X_{n}^{(i v)}(x) T_{n}(t)+\rho A X_{n}(x) T_{n}^{\prime \prime}(t)=-M g \delta(x-v t)$

Applying the Finite Fourier Sine Transform in (39) and (40) Where
We have

$f_{n}(t)=-2 g \int_{0}^{L} X_{n}(x) \delta x-2 \frac{M}{L} X_{n}(v t) \int_{0}^{L}\left(\begin{array}{c}X_{n}(x) T_{n}^{\prime \prime}(t)+2 v X_{n}(x) T_{n}^{\prime}(t) \\ +v^{2} X_{n}^{\prime \prime}(x) T_{n(t)}\end{array}\right) \delta(x-v t)$

Substituting (41) and (42) into (11), we have

$\bar{F}(x, t)=\left[-2 g \int_{0}^{L} X_{n}(x) \delta x\right.$

$$
-2 \frac{M}{L} X_{n}(v t) \int_{0}^{L}\left(X_{n}(x) T_{n}^{\prime \prime}(t)+2 v X_{n}^{\prime}(x) T_{n}^{\prime}(t)+\right.
$$

$$
\left.\left.v^{2} X_{n}^{\prime \prime}(x) T_{n}(t)\right) \delta(x-v t)\right] \sin \frac{n \pi}{L}
$$

Since $X_{n}(x)=\sin \frac{n \pi}{L} x$ we then have

$$
X_{n}(v t)=\sin \frac{n \pi v}{L} x
$$

Substituting (43) and (44) into (40), we have

$E I\left(\frac{n \pi}{L}\right)^{4} \sum_{n=1}^{\infty} T_{n}(t) \sin \frac{n \pi}{L} x+\rho A \sum_{n=1}^{\infty} T_{n}^{\prime \prime}(t) \sin \frac{n \pi v}{L}$

$=\sum_{n=1}^{\infty}\left[-2 g \frac{M}{L} \sin \frac{n \pi v}{L} t-2 \frac{M}{L}\left(\begin{array}{c}\sin \frac{n \pi v}{L} t T_{n}^{\prime}(t)+2 v \frac{n \pi}{L} \cos \frac{n \pi v}{L} t \sin \frac{n \pi v}{L} t T_{n}^{\prime \prime}(t) \\ -\left(\frac{n \pi}{L}\right)^{2} v^{2} \sin \frac{n \pi v}{L} t \sin \frac{n \pi v}{L} t \sin \frac{n \pi v}{L} t T_{n}(t)\end{array}\right)\right] \sin \frac{n \pi}{L} x$

Divide both sides by $\quad \sum_{n=1}^{\infty} \sin \frac{n \pi}{L} x$

We then have $T_{n}^{\prime \prime}(t)+Q_{1} T^{\prime}(t)+Q_{2} T_{n}(t)=Q_{3} \sin \bar{\omega}_{n}(t)$

Where

$Q_{1}=\frac{H_{5}}{H_{2}+H_{4}}, \quad Q_{2}=\frac{H_{1}-H_{6}}{H_{2}+H_{4}}, \quad Q_{3}=\frac{H_{3}}{H_{2}+H_{4}}, \quad H_{1}=E I\left(\frac{n \pi}{L}\right)^{4}, \quad H_{2}=\rho A, \quad H_{3}=-2 g \frac{M}{L}$,

$\bar{\omega}_{n}=\frac{n \pi v}{L}, \quad H_{4}=2 \frac{M}{L} \sin \frac{n \pi v}{L} t \sin \frac{n \pi v}{L} t, \quad H_{5}=4 M v \frac{n \pi}{L^{2}} \cos \frac{n \pi v}{L} t \sin \frac{n \pi v}{L} t$,

Solving the homogeneous part of (46), we obtained solution of the form

$m_{1,2}=-\frac{Q_{1}}{2} \pm \sqrt{\left(\frac{Q_{1}}{2}\right)^{2}-Q_{2}}$

$\mathrm{A}_{\mathrm{SN}}$

P-ISSN 2536-6904, E-ISSN 2705-2761 African Journal of Science \& Nature Vol. 10, 88-96 (2020) 
Using the method of undetermined coefficient, let

$T_{p}(t)=F_{n} \sin \bar{\omega}_{n} t+G_{n} \cos \bar{\omega}_{n} t$

Differentiating (48), we have

$$
G_{n}=\frac{Q_{3} \omega_{n} Q_{1}}{\left(\bar{\omega}_{n}^{2}-Q_{2}\right)\left(Q_{2}-\bar{\omega}_{n}^{2}\right)-\bar{\omega}_{n}^{2} Q_{1}^{2}}
$$

and

$$
F_{n}=\frac{Q_{3} \omega_{n} Q_{1}\left(\bar{\omega}_{n}^{2}-Q_{2}\right)}{\omega_{n} Q_{1}\left(\bar{\omega}_{n}^{2}-Q_{2}\right)\left(Q_{2}-\bar{\omega}_{n}^{2}\right)-\bar{\omega}_{n}^{3} Q_{1}^{3}}
$$

So that

$T_{p}(t)=\frac{Q_{8} \omega_{n} Q_{1}\left(\bar{\omega}_{n}^{2}-Q_{2}\right)}{\omega_{n} Q_{1}\left(\bar{\omega}_{n}^{2}-Q_{2}\right)\left(Q_{2}-\bar{\omega}_{n}^{2}\right)-\bar{\omega}_{n}^{8} Q_{1}^{8}} \sin \bar{\omega}_{n} t+\frac{Q_{8} \omega_{n} Q_{1}}{\left(\bar{\omega}_{n}^{2}-Q_{2}\right)\left(Q_{2}-\bar{\omega}_{n}^{2}\right)-\bar{\omega}_{n}^{2} Q_{1}^{2}}$

Hence, we have

$T_{n}(t)=A e^{m_{1} t}+B e^{m_{2} t}+\bar{R}_{n}\left(K_{n} \sin \bar{\omega}_{n} t+\cos \bar{\omega}_{n} t\right)$

Where

$$
R_{n}=\frac{Q_{3} \omega_{n} Q_{1}\left(\bar{\omega}_{n}^{2}-Q_{2}\right)}{\omega_{n} Q_{1}\left(\bar{\omega}_{n}^{2}-Q_{2}\right)\left(Q_{2}-\bar{\omega}_{n}^{2}\right)-\bar{\omega}_{n}^{3} Q_{1}^{3}}, \quad K_{n}=\frac{\left(\bar{\omega}_{n}^{2}-Q_{2}\right)}{\omega_{n} Q_{1}}
$$

Substituting the expression for $T_{n}(t)$ and $T_{n}^{\prime}(t)$ and then solving it, we get $\sum_{n=1}^{\infty} T_{n}(0) \sin \frac{n \pi}{L} x=0$

and

$\sum_{n=1}^{\infty} T_{n}^{\prime}(0) \sin \frac{n \pi}{L} x=0$

$T_{n}(t)=A_{n} e^{m_{1} t}+B_{n} e^{m_{2} t}+\bar{R}_{n}\left(K_{n} \sin \bar{\omega}_{n} t+\cos \bar{\omega}_{n} t\right)$

$T_{n}^{\prime}(t)=T_{n}(t)=A_{n} e^{m_{1} t}+B_{n} e^{m_{2} t}+\bar{R}_{n}\left(K_{n} \sin \bar{\omega}_{n} t+\cos \bar{\omega}_{n} t\right)$

Substituting the expression $T_{n}(t)$ and $T_{n}^{\prime}(t)$ and then solving it, we get

$B_{n}=\frac{R_{n}\left(m_{1}-K_{n}\right)}{\left(m_{1}-m_{2}\right)}$

$A_{n}=\frac{R_{n}\left(K_{n}-m_{1}\right)}{\left(m_{1}-m_{2}\right)}$
$T_{n}(t)-\frac{\bar{R}_{n}}{\left(m_{1}-m_{2}\right)}\left[\left(K_{n}-m_{2}\right) e^{m_{1} t}+\left(m_{1}-K_{n}\right) e^{m_{2} t}+\bar{R}_{n}\left(K_{n} \sin \bar{\omega}_{n} t+\cos \bar{\omega}_{n} t\right)\right]$

$W(x, t)=\sum_{n=1}^{\infty}\left\{\frac{\bar{R}_{n}}{\left(m_{1}-m_{2}\right)}\left[\left(K_{n}-m_{2}\right) e^{m_{1} t}+\left(m_{1}-K_{n}\right) e^{m_{2} t}\right.\right.$

$$
\left.\left.+\bar{R}_{n}\left(K_{n} \sin \bar{\omega}_{n} t+\cos \bar{\omega}_{n} t\right)\right]\right\} \sin \frac{n \pi}{L} x
$$

Equation (60) is the deflection of the beam subjected to moving mass.

\section{RESULTS}

MATLAB was used and thenumerical data,given below, were used

$\rho A=0.98 \mathrm{kgm}^{-1}, \quad E=2.10 \times 10^{11} \mathrm{~N}, \quad b=0.001 \mathrm{~m}, \quad d=0.0001 \mathrm{~m}, \quad M=50,100 \mathrm{~kg}$,

$L=6 \mathrm{~m}, \quad m=7 \mathrm{~kg}, \quad v=28 \mathrm{~m} / \mathrm{s}$

Figures 1-4 show the deflection profiles of the beam depicted graphically to demonstrate the effect of the velocity of the load, time at which the load moves and increase in mass of the load.

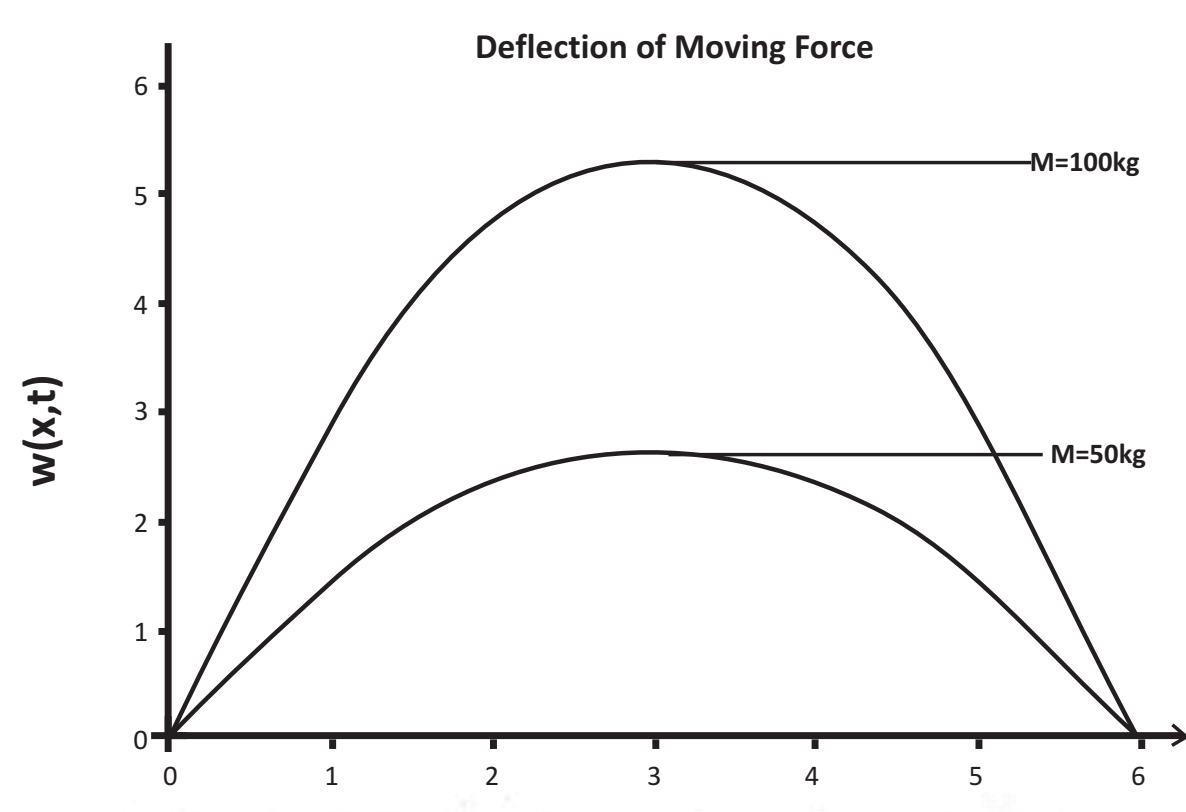

Figure 1: Deflection of beam subjected to moving force 


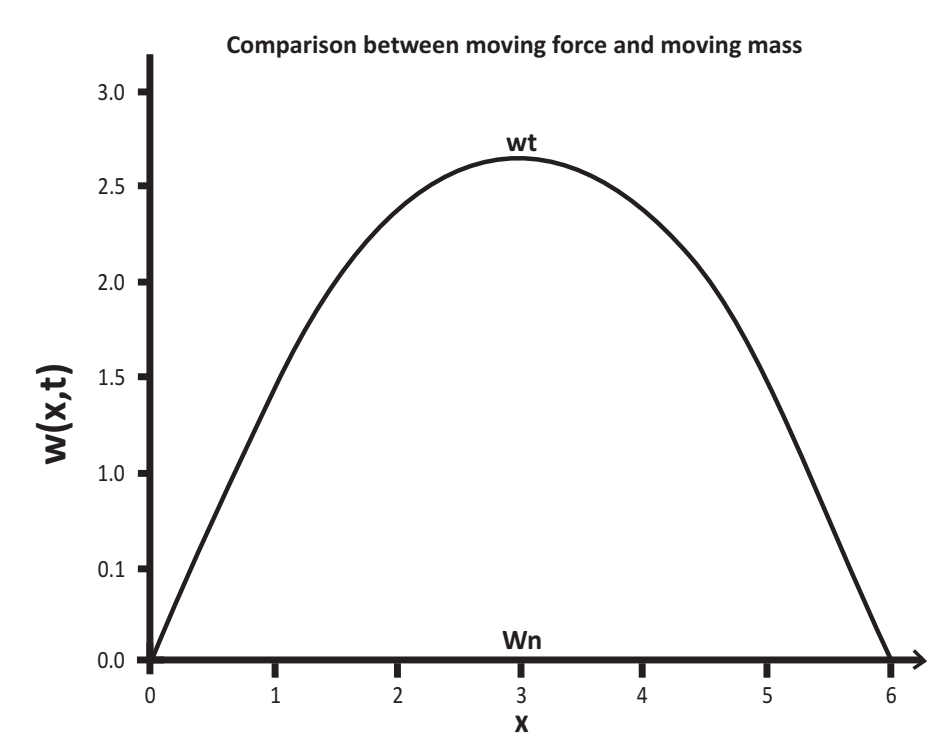

Figure 3: Comparison between moving force and moving mass for $\mathrm{M}=59 \mathrm{~kg}$

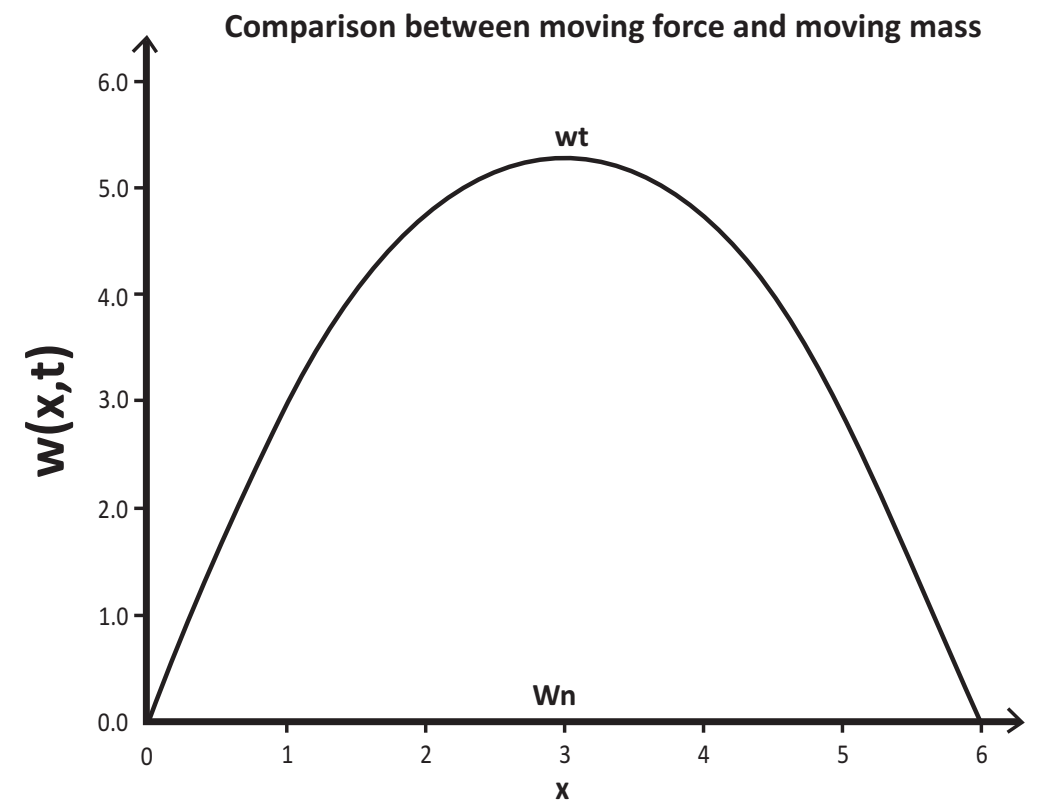

Figure 4: Comparison between moving force and moving mass for $\mathrm{M}=100 \mathrm{~kg}$.
Awodola T.O (2005): Influence of foundationand axial force on the vibration of thin beam undervariable harmonic moving load. Journal of the Nigerian Association of Mathematical Physics (JNAMP) 9:143-150

Chen and Jien (2011) Dynamic response ofclamped axially moving beams: integral TransformSolution. Applied Mathematics and Computation 218:249-259

Gbadeyan J A and Idowu A.S (2002): Theresponse of a pre-stressed Bernoulli beam carrying anadded mass to a number of concentrated movingloads. Abacus Journal of Mathematical Association of Nigerian 29:101-110.

Gbadeyan J.A and Usman M.A. (2003):Vibrational analysis of Euler-Bernoulli beam withviscous damping coefficient subjected to distributedmoving load. Nigeria Journal of Pure and Applied science 18:1492-1500.

Idowu A.S., Dada M.S. and Titiloye E.O. (2004):The response of pre-stressed Bernuolli beamtransversed by partially distributed moving loads.Abacus Journal of Mathematical Association of Nigerian 31(2A):17-27.

Ismail E. (2011); Dynamic response of a beamdue to an accelerating moving mass using movingfinite element approximation. Association for Scientific Research, Mathematical and Computational Application 16(1):171-182.

Kozien M.S. (2013): Analytical solution ofexcited vibrations of a beam with application ofdistribution, Acta Physics Polonics A.
123(6):1029-1033.

Lu S. and Freiguan L. (2008): Steady statedynamic response of a Bernoulli-Euler beam on aviscoelastic foundation subjected to a moving platoonof moving loads. Journal of Vibration and Acousticsvol 130/051002:119

Naguleswan S. (2006): Vibration of EulerBernoulli uniform beam carrying a rigid body at eachend. International Journal of Mechanical Engineering Education 34(3): Mechanical.

Omolafe B. and Ogunyebi S.N. (2009):Transvers vibrations of elastic thin beam resting onvariable resting on variable elastic foundations andsubjected to traveling distributed forces. The Pacific journal of Science and Technology 10(2):112-119.

Usman M.A. (2003): Dynamic response of aBernoulli beam on winkler foundation under theaction of moving partially distributed moving load.Nigeria Journal of Mathematics and Application 16:128-147.

Usman, M.A and Muibi, B.T (2019): Effect of Free Vibration Analysis on Euler-Bernoull Beam with different Boundary Conditions. 3(4), 501-510. FUDMA Journal of Science (FJS)

Usman, M.A and Adeboye, O.A (2019). Mathematical Analysis of Euler-Bernoulli Beam with Damping Coefficient Subjected to Moving Load. Annal Computer Journal Series. Anale Series Informatica XVII fasc 2

\section{DISCUSSION AND CONCLUSION}

The response of vibration analysis of beam subjected to moving force and moving mass was investigated. Finite Fourier Sine transform with undetermined coefficient was used to solve the governing equation.

From the response profile of the beam, it was found that the response amplitude increases as the mass of the load increases for the case of moving force while the response amplitude for the case of moving mass is not affected by increase in mass of the load. Also analysis shows that the response than that of moving mass.

\section{ACKNOWLEGMENTS}

The authors wish to express their deepest gratitude to God for His guidance throughout the research.

\section{REFERENCES}

Abu-Hilal M. (2003): Forced vibration of EulerBernoulli beams by means of dynamic greenfunction. Academic Press, Journal of Sound and Vibration 267:191-207.

Akinpelu F.O (2012): The response of viscouslydamped Euler-Bernoulli Beam to uniform partiallydistributed moving loads. Applied Mathematics, Scientific Research, 3:199-204 